\title{
Principal Sensory Nucleus of the Trigeminal Nerve
}

National Cancer Institute

\section{Source}

National Cancer Institute. Principal Sensory Nucleus of the Trigeminal Nerve. NCI

Thesaurus. Code C33402.

A collection of second order neurons, located in the pons lateral to the trigeminal motor nucleus and medial to the middle cerebellar peduncle, that receive and relay data regarding both light touch and discriminative sensation of the face, as well as conscious proprioception of the jaw. 